\title{
Palisade pigmentation - describing a new dermoscopic finding
}

\section{Subrata Malakar, Anjali Pal, Pratibha Pradhan}

\author{
Rita Skin Foundation, Kolkata, India
}

Corresponding author: Dr. Anjali Pal, E-mail: unjelly@gmail.com

Sir,

Discoid lupus erythematosus (DLE) represents the most common subtype of cutaneous lupus erythematosus. There is a morphological diversity seen in DLE, at different stages of progression of the disease. Based on these features, dermoscopic criteria have been formed for both early and late stage DLE, and their histopathological correlations studied [1]. We would like to describe a new dermoscopic feature that we have observed in late stage DLE- palisade pigmentation.

Case 1: A 26year old male presented with a lesion over the right pre-auricular area, of nine months duration. Examination revealed an irregularly shaped, depigmented atrophied plaque with a central erythematous area and overlying adherent scales, hyperpigmented border, and absence of hair follicles (Fig. 1). Dermoscopy revealed white shiny structures, telangiectasia, white scales, and palisade pigmentation (Fig. 2).

A biopsy from the lesion showed features confirming the diagnosis of DLE (Fig. 3).

Case 2: A 35year old man noticed multiple lesions on his right cheek, over the past six months. They were asymptomatic. Cutaneous examination revealed multiple irregular violaceous atrophic plaques on the right cheek, with an erythematous border. Dermoscopy revealed white shiny structures, thick white overlying scales, telangiectasia, and palisade pigmentation (Fig. 4). Biopsy confirmed the diagnosis of DLE.

A 'palisade' is a fence of wooden stakes or iron railings fixed in the ground, forming an enclosure or defence. In dermatopathology, the term has been used to describe the typical arrangement of basaloid cells in basal cell carcinoma, and in granuloma annulare, for histiocytes surrounding an area of altered collagen [2].

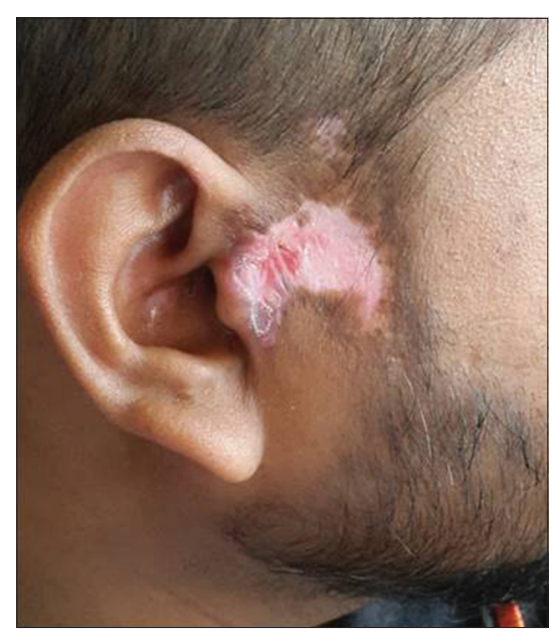

Figure 1: Depigmented atrophied plaque with scaling, over the right pre-auricular area.

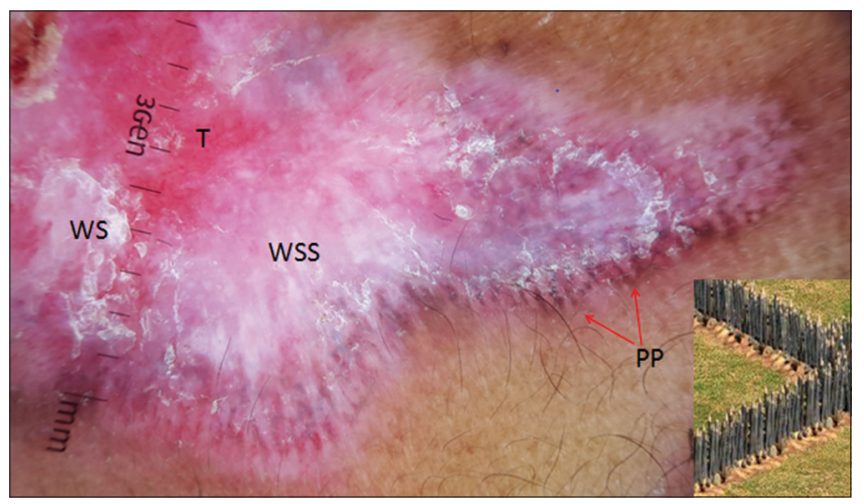

Figure 2: Palisade pigmentation (PP) seen on dermoscopy of the lesion. Note other features of the disease such as white shiny structures (WSS), telangiectasis (T) and white scales (WS), which are findings in late stage DLE. Inset shows a picture of palisade arrangement.

\footnotetext{
How to cite this article: Malakar S, Pal A, Pradhan P. Palisade pigmentation - describing a new dermoscopic finding. Our Dermatol Online. 2018;10(1):106-107. Submission: 24.04.2018; Acceptance: 19.06.2018

DOI:10.7241/ourd.20191.32
} 


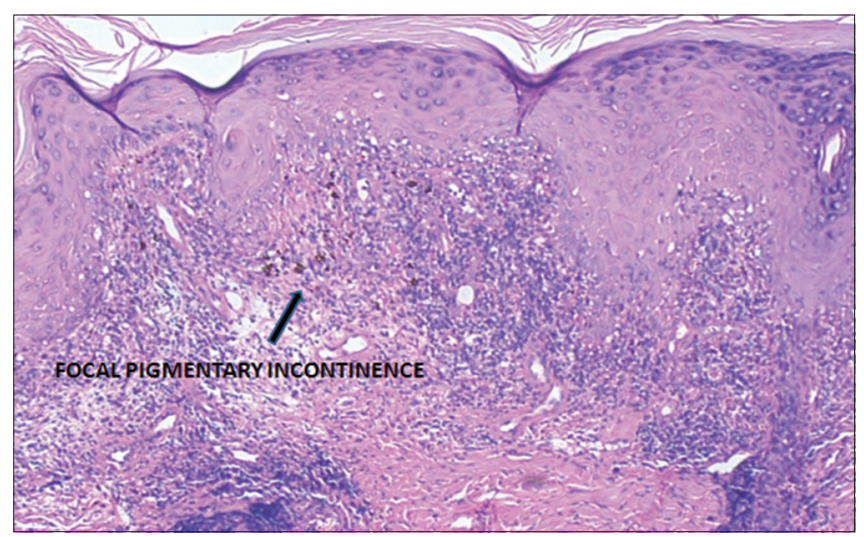

Figure 3: Focal pigmentary incontinence on histopathology corresponds to palisade pigmentation on dermoscopy.

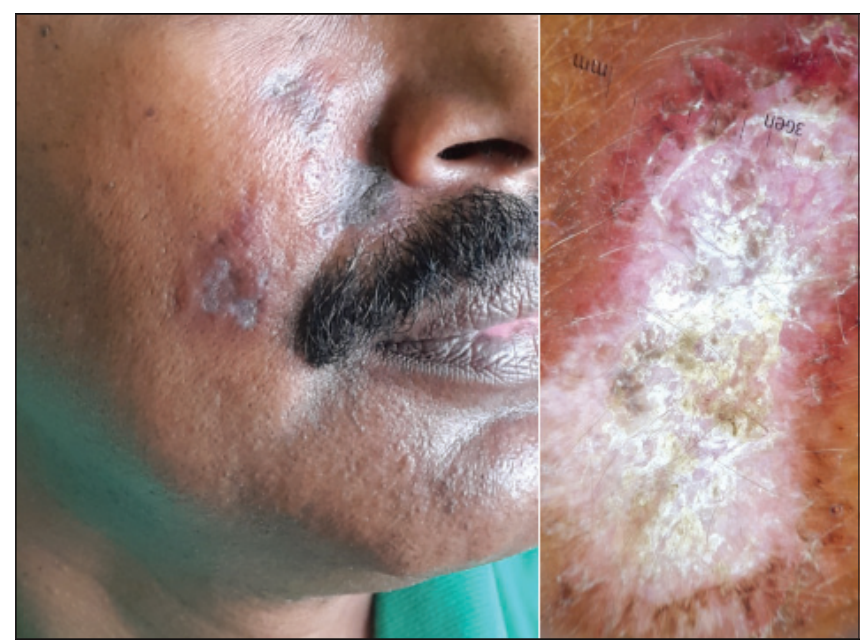

Figure 4: Erythematous plaques with a violaceous tinge, on the right cheek. Dermoscopy showing white shiny structures, thick white scales and palisade pigmentation.

'Palisade pigmentation' observed on dermoscopy here, is formed by the arrangement of pigment structures at the periphery of the lesion in late stage DLE. It appears as linear hyperpigmented streaks. They may be present focally or segmentally, and may extend for a distance, outwards, from the periphery of the lesion.
Histopathologically, it corresponds to pigmentary incontinence, which was confirmed in our case. The incontinence may not involve the entire dermis, but is present focally or segmentally, and is due to focal interface vacuolar degeneration of the basal layer.

Hyperpigmentation has been described in dermoscopy of late stage DLE $[1,3]$ in the form of honeycomb network, perifollicular pigmentation, radial pigment streaks or pigmentation arranged in unspecified patterns. In a study by Lallas et al [1], hyperpigmentation was observed on dermoscopy in $43.6 \%$ cases of DLE, and pigmentary incontinence was seen in $49.1 \%$ of the corresponding biopsies.

We would like to suggest this characteristic finding of 'palisade pigmentation' as a dermoscopic indicator of late stage DLE. Recognition of this pattern, in conjunction with other already established dermoscopic features, can help clinch the diagnosis.

\section{CONSENT}

The examination of the patient was conducted according to the Declaration of Helsinki principles.

\section{REFERENCES}

1. Lallas A, Apalla Z, Lefaki I, Sotiriou E, Lazaridou E, Ioannides D, et al. Dermoscopy of discoid lupus erythematosus. Br J Dermatol. 2013;168:284-8.

2. Madke B, Chougule BD, Kar S, Khopkar U. Appearances in clinical dermatology. Indian J Dermatol Venereol Leprol. 2014;80:432-47.

3. Errichetti E, Stinco G. Dermoscopy in General Dermatology: A Practical Overview. Dermatol Ther. 2016;6:471-507.

Copyright by Subrata Malakar, et al. This is an open-access article distributed under the terms of the Creative Commons Attribution License, which permits unrestricted use, distribution, and reproduction in any medium, provided the original author and source are credited.

Source of Support: Nil, Conflict of Interest: None declared. 